\title{
Correction: Correlation between cognition and plasma noradrenaline level in Alzheimer's disease: a potential new blood marker of disease evolution
}

\author{
Laure-Elise Pillet, Camille Taccola, Justine Cotoni, Hervé Thiriez, Karine André and Romain Verpillot
}

Correction to: Translational Psychiatry https://doi.org/10.1038/s41398-020-0841-7, published online 3 July 2020

We have updated this Article since its original publication in order to change a sentence in the Acknowledgements section. Originally, the sentence relating to Alzohis and Pr. Claire Paquet read:

"We also thank Claire Paquet, MD, PhD (Memory Resources and Research Center, Cognitive Neurology Center, INSERM UMR-S 942, University Hospital of Paris Diderot Saint Louis-Lariboisière-Fernand Widal, APHP, France) for initiating and conducting this study as well as Jacques Callebert, PharmD, PhD (Department of Biochemistry, University Hospital of Paris Diderot Saint
Louis-Lariboisière-Fernand Widal, APHP, France) for performing the blood sample analyses".

We've changed this to:

"We also thank Claire Paquet, MD, Ph.D. (Memory Resources and Research Center, Cognitive Neurology Center, INSERM UMR-S 942, University Hospital of Paris Diderot Saint Louis-Lariboisière-Fernand Widal, APHP, France) for providing the biological and clinical samples as well as Jacques Callebert, PharmD, Ph.D. (Department of Biochemistry, University Hospital of Paris Diderot Saint Louis-Lariboisière-Fernand Widal, APHP, France) for performing the blood sample analyses".

Published online: 24 November 2020 